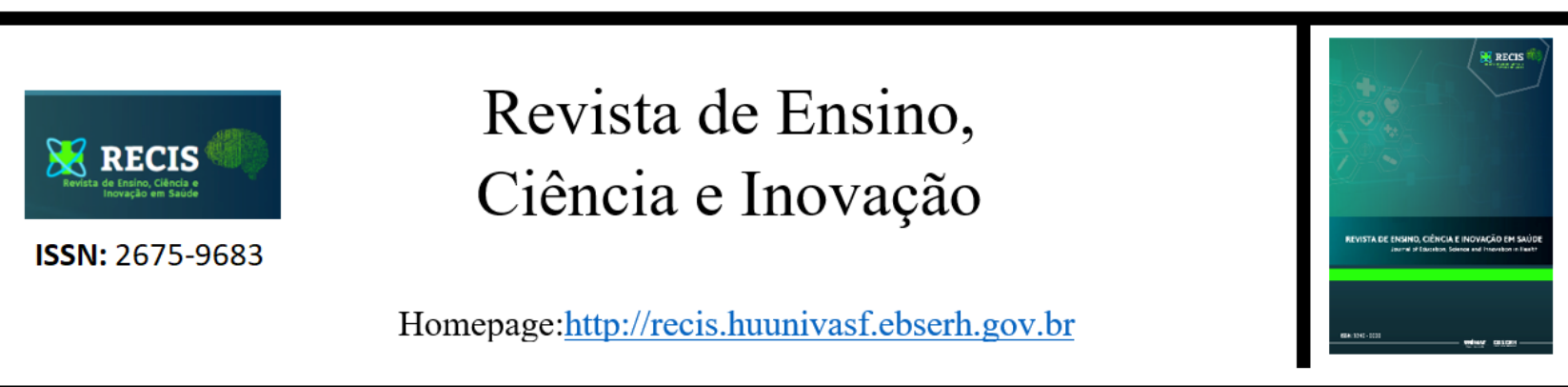

\title{
Perfil dos Pacientes Vítimas de Trauma Torácico Submetidos à Drenagem de Tórax Atendidos em um Hospital Universitário
}

\section{Profile of Thoracic Trauma Patients Undergoing Chest Drainage Attended at a University Hospital}

\section{Ester Menezes Silva Bonfim ${ }^{1}$, Diva Danielly Rêgo de Vasconcelos ${ }^{1}$, Lílian Ramine Ramos de Souza Matos ${ }^{1}$}

${ }^{1}$ Fisioterapeuta, Hospital Universitário da Universidade Federal do Vale do São Francisco

Autor correspondente: ester.bonfim@ebserh.gov.br

Artigo recebido em 20/12/2020 e aceito em 12/04/2021

\begin{abstract}
RESUMO
O trauma torácico é uma importante causa de morte evitável, acometendo principalmente os jovens do sexo masculino de 20 a 30 anos. O objetivo deste estudo é traçar o perfil dos pacientes vítimas de trauma torácico submetidos à drenagem de tórax. Estudo observacional, com abordagem quantitativa e exploratória, apresentação descritiva e caráter documental e retrospectivo, através do acesso autorizado ao software do Aplicativo de Gestão para Hospitais Universitários (AGHU) e da coleta de dados dos prontuários dos pacientes da Clínica Cirúrgica do Hospital Universitário da Universidade Federal do Vale do São Francisco em Petrolina-PE (HU-UNIVASF) no período de maio de 2017 a março de 2018. Foram encontrados 82 homens $(89,13 \%)$ e 10 mulheres $(10,86 \%)$, a maioria entre 21 e 40,8 anos. O tempo de drenagem torácica foi entre três e sete dias, os ferimentos por arma branca $(56,5 \%)$ e o tipo de lesão hemopneumotórax $(42,4 \%)$ foram mais frequentes. As lesões ocorreram mais no hemitórax esquerdo (56,5\%). Foi concluído que o perfil das vítimas de trauma torácico submetidos à drenagem de tórax internados no HU-UNIVASF foi formado por homens jovens, com lesão decorrente de ferimento por arma branca, levando a hemopneumotórax, sendo o hemitórax esquerdo mais acometido, resultando em um tempo médio de quatro dias com dreno de tórax.

Palavras-chave: Perfil de Saúde; Traumatismos torácicos; Lesões torácicas; Drenagem por sucção.
\end{abstract}

\begin{abstract}
Chest trauma is an important cause of preventable death, affecting mainly young men aged 20 to 30 years. The objective is to outline the profile of patients suffering from chest trauma who underwent chest drainage. Observational study, with a quantitative and exploratory approach, descriptive presentation and documentary and retrospective character, through authorized access to the software of the Management Application for University Hospitals (AGHU) and the collection of data from the medical records of patients at the Surgical Clinic of the University Hospital of the University Federal District of the São Francisco Valley in Petrolina-PE (HU-UNIVASF) from May 2017 to March 2018. 82 men (89.13\%) and 10 women $(10.86 \%)$ were found, most of them between 21 and 40.8 years. Chest drainage time was between three and seven days, stab wounds (56.5\%) and type of hemopneumothorax lesion $(42.4 \%)$ were more frequent. The lesions occurred more in the left hemithorax $(56.5 \%)$. We conclude that the profile of chest trauma victims who underwent chest drainage admitted to the HU-UNIVASF was formed by young men, with injuries resulting from stab wounds, leading to hemopneumothorax, with the left hemithorax being more affected, resulting in an average time for four days with a chest drain.
\end{abstract}

Keywords: Health Profile; Thoracic injuries; Thoracic injuries; Suction drainage. 


\section{INTRODUÇÃO}

O trauma em geral é uma das principais causas de invalidez e morte no mundo, vem apresentando uma tendência de aumento nos últimos anos e se constitui como a terceira causa de morte na população e a maior em indivíduos menores de 40 anos. O trauma torácico é uma importante causa de morte evitável, acometendo principalmente os jovens do sexo masculino de 20 a 30 anos. As lesões podem ser decorrentes de acidentes automobilísticos (particularmente com motocicletas), atropelamentos e quedas classificados de trauma fechado e decorrentes de ferimentos intencionais com armas branca e de fogo, classificados como trauma aberto/penetrante e conforme a região estudada, podem mudar a frequência com que ocorrem. ${ }^{1}$

Em 2013, no Brasil, segundo dados do Sistema de Informação sobre Mortalidade do Ministério da Saúde do Brasil (SIM/MS), as causas externas, que incluem as violências e os acidentes, são a terceira causa de morte na população em geral e a primeira causa na faixa etária de 15 a 39 anos, sendo que $91,7 \%$ das vítimas eram do sexo masculino, $60,7 \%$ da raça parda. ${ }^{2}$

Em 2010, no estado de Pernambuco, as causas externas ocuparam a segunda posição (14,6\% dos óbitos), com destaque para os provocados por acidentes de transporte terrestre. Neste mesmo estudo, houve o destaque da VIII Gerência Regional de Saúde - VIII Geres (a qual Petrolina está inserida) para o aumento de óbitos por acidentes. ${ }^{3}$

Os traumas torácicos apresentam considerável gravidade, pois para cada quatro vítimas de trauma que morrem, uma tem lesão no tórax como causa de óbito. ${ }^{4}$

O sucesso neste tipo de atendimento exige um diagnóstico precoce e tratamento imediato e deve ser realizado pelos profissionais com rapidez, organização e com eficiência. Uma conduta adequada é baseada nos princípios do suporte de vida avançado em trauma (ATLS). O Colégio Americano de Cirurgiões criou o mnemônico ABCDE no sentido de padronizar o atendimento ao politraumatizado, identificando lesões potencialmente fatais ao indivíduo e o seu significado é: A (airways) - vias aéreas com controle da coluna cervical; B (breathing) respiração e ventilação; C (circulation) circulação com controle da hemorragia; D (disability) - estado neurológico; E (exposure) - exposição e controle da temperatura. Procedimento simples, mas que é decisivo para a sobrevida do paciente. $^{5}$

A maior parte dos traumas torácicos é representada por pneumotórax, hemotórax ou hemopneumotórax prontamente tratados com drenagem pleural e somente 15 a $30 \%$ destes necessitarão de uma toracotomia. ${ }^{6} \mathrm{~A}$ drenagem de tórax é um procedimento cirúrgico aparentemente simples que visa promover a saída contínua de ar ou líquido, permitindo o restabelecimento das pressões negativas no espaço pleural. $^{7} \mathrm{O}$ dreno torácico é conectado a um sistema chamado selo d'água que consiste em uma válvula unidirecional que impede a entrada de ar para o interior do espaço pleural durante a inspiração. É um sistema eficiente, seguro e de baixo custo. ${ }^{8}$

Diante dessas informações, fortalecemos a importância da realização desta pesquisa neste hospital universitário, que tem como objetivo conhecer o perfil dos pacientes vítimas de traumatismo torácico submetidos à drenagem de tórax.

\section{MATERIAL E MÉTODOS}

Trata-se de um estudo observacional, com abordagem quantitativa e exploratória, apresentação descritiva e caráter documental e retrospectivo, através do acesso autorizado ao software AGHU e da coleta de dados dos prontuários de pacientes admitidos por trauma torácico e submetidos a drenagem pleural em selo d'água. Este estudo foi realizado na Clínica Cirúrgica do Hospital Universitário da Universidade Federal do Vale do São Francisco em Petrolina-PE (HU-UNIVASF) no período de maio de 2017 a março de 2018, obedecendo a Resolução do Conselho Nacional de Saúde (CNS) No466/2012, obtendo aprovação pelo Comitê de Ética em Pesquisa (CEP) sob N ${ }^{0}$ 2.769.009.

Foram incluídos na pesquisa todos os prontuários de pacientes internados na enfermaria da Clínica Cirúrgica com drenagem torácica, somente em consequência de trauma torácico, com ou sem traumatismos orgânicos múltiplos, de ambos os sexos e de qualquer idade. Não foram incluídos os prontuários de pacientes internados nesta enfermaria com drenagem torácica por outras causas que não o trauma e aqueles que tiveram tratamento conservador. Os pacientes excluídos foram os que receberam alta hospitalar com tubostomia, os que foram a óbito no setor durante 
a pesquisa e os que foram transferidos para outro setor.

Uma ficha estruturada pelos pesquisadores compilou os seguintes dados: gênero, idade, tempo de permanência com a drenagem, mecanismo da lesão, o tipo de lesão e o lado do tórax acometido.

Este estudo analisou 92 pacientes vítimas de trauma torácico que foram submetidos à drenagem de tórax. Estes pacientes receberam atendimento fisioterapêutico e foram acompanhados até a alta hospitalar, sendo monitorizada a evolução do dreno torácico através dos critérios de retirada como: avaliação radiológica da expansão pulmonar, débito do dreno até $100 \mathrm{ml} / 24$ horas, aspecto do volume drenado e ausência de escape aéreo. $\mathrm{O}$ atendimento fisioterapêutico era iniciado assim que o paciente chegava ao setor, objetivando favorecer a drenagem torácica, a reexpansão pulmonar e prevenção de possíveis complicações pleuropulmonares.

Os dados foram processados e analisados utilizando os programas Statistical Package for the Social Sciences - SPSS (SPSS Inc., Chicago, IL, EUA, Release 16.0.2, 2008). Após consolidação e validação dos dados inseridos foi utilizada a estatística descritiva. Variáveis contínuas foram apresentadas em valores de média e desvio padrão após verificação da normalidade por meio do teste de Kolmogorov-Smimov, enquanto as variáveis categóricas estão apresentadas em frequências absoluta e relativa. Todas as análises foram bicaudais, valores de $\mathrm{p}$ quando significativos foram calculados e $5 \%$ foi o nível de significância adotado.

\section{RESULTADOS}

O perfil dos pacientes incluídos na análise foi composto pela maioria do gênero masculino $(\mathrm{n}=82,89,13 \%)$, com uma média de idade de 31,6 anos $( \pm 12,7)$. Em relação ao tempo com o dreno de tórax, houve uma média de 5,8 dias $( \pm 5,1)$.

Quanto ao mecanismo da lesão, 56,5\% $(n=52)$ dos casos foram causados por perfuração de arma branca com $n=52$ totalizando $56,5 \%$. Os traumas ocasionaram em $42,4 \% \quad(n=39)$ dos indivíduos o hemopneumotórax, nos quais a lateralidade mais acometida foi o hemitórax esquerdo (56,5\%, $\mathrm{n}=52)$. Conforme detalha (Tabela 1).
Tabela 1: Caracterização dos mecanismos e tipos de lesão de trauma torácico.

\begin{tabular}{lcc}
\hline Variáveis & $\mathbf{n}$ & $\mathbf{\%}$ \\
\hline Mecanismo de lesão & & \\
Acidente automobilistico & 14 & 15,2 \\
Agressão física ou queda & 3 & 3,3 \\
Atropelamento & 5 & 5,4 \\
Perfuração por arma branca & 52 & 56,5 \\
Perfuração por arma de fogo & 18 & 19,6 \\
\hline Tipos de lesão & & \\
Derrame pleural & 2 & 2,2 \\
Hemopneumotorax & 39 & 42,4 \\
Hemotorax & 32 & 34,8 \\
Pneumotorax & 17 & 18,5 \\
Mais de uma lesão & 2 & 2,2 \\
\hline Hemitórax & & \\
HTD & 33 & 35,9 \\
HTE & 52 & 56,5 \\
HTD/HTE & 7 & 7,6 \\
\hline
\end{tabular}

HTD: Hemitórax Direito. HTE: Hemitórax Esquerdo. Quanto a comparação do tempo de dreno e o tipo de lesão, não houve diferença estatisticamente significativa $(\mathrm{p}>0,05)$.

\section{DISCUSSÃO}

Os resultados obtidos nessa pesquisa corroboram com os achados de outros estudos em que houve predomínio de adultos jovens do gênero masculino, ${ }^{1,11}$ mostrando-se bem coerente ao que é apresentado na literatura. Devido à violência urbana, as agressões físicas são mais frequentes através de disputas entre grupos de rua ou em bares, afetando mais os indivíduos do sexo masculino, que são seus maiores frequentadores. ${ }^{6}$

A região onde foi realizado o presente estudo não é diferente, existe a violência urbana e somado a isso o uso de bebida alcóolica, principalmente nos finais de semana, que só exacerba o fato.

Quanto ao período com o dreno torácico, muitos autores defendem que o tempo máximo de permanência do dreno na cavidade pleural é de três a oito dias. ${ }^{10}$ No presente estudo, os pacientes permaneceram entre três e sete dias, concordando com a literatura. No setor da Clínica Cirúrgica do HU-UNIVASF os pacientes realizam fisioterapia, a qual é realizada seguindo protocolo assistencial realizado pela equipe.

No Brasil, grande parte dos casos de trauma torácico ocorrem devido à violência urbana 
e com predomínio dos ferimentos penetrantes causados por arma branca ou de fogo. ${ }^{6,9}$

No presente estudo a situação não se mostrou diferente, pois o mecanismo de lesão mais encontrado foi por perfuração de arma branca. Podemos também comprovar tais informações com os achados em um estudo realizado em um hospital da rede pública municipal de São Luís, no Maranhão, que demostrou que a causa mais comum do trauma torácico foi o ferimento por arma branca. ${ }^{10}$ Possivelmente a justificativa para isso seja porque as regiões mais pobres como o nordeste, utilizam-se de facas, pois estas têm menor custo em relação às armas de fogo. ${ }^{6}$ A cidade de Petrolina, faz parte do Sertão Pernambucano, e também compartilha da mesma realidade, onde as armas brancas são mais baratas e mais acessíveis.

Quanto ao tipo de lesão, esta pesquisa evidenciou um maior número de casos de hemopneumotórax o que só fortalece que o trauma torácico exige um diagnóstico rápido e intervenção imediata, sendo necessária uma avaliação rápida das condições clínicas do paciente, enfatizando a oxigenação, a presença ou não de choque hipovolêmico e após a drenagem torácica, observar a sua eficácia. ${ }^{10}$ Divergindo dos resultados deste estudo, em que o hemopneumotórax foi mais frequente, um estudo realizado em um hospital de urgência e emergência da Amazônia Legal encontrou mais hemotórax (33\%) e pneumotórax $(33 \%)$ separadamente como tipos de lesões mais frequentes. $^{12}$

Referente ao hemitórax acometido, neste estudo o destaque foi para o hemitórax esquerdo. Este dado concorda com um estudo ${ }^{13}$ que relata que apesar do mecanismo do trauma ter sido fechado por colisão, o hemitórax acometido foi o esquerdo com 40,8\% dos pacientes, o hemitórax direito com $38,7 \%$ e drenagem bilateral com 18,4\%. Provavelmente, tenha alguma relação dos ferimentos penetrantes serem de causa intencional e o hemitórax esquerdo ser considerado como uma região com maior probabilidade de ser fatal quando lesionada.

\section{LIMITAÇÕES DO ESTUDO}

O tempo de coleta poderia ter sido estendido para que um número de amostras fosse maior e assim realizar a estatística analítica, extrapolando os resultados para a população geral.

\section{CONCLUSÃO}

O perfil das vítimas de trauma torácico submetidos à drenagem de tórax internados na Clínica Cirúrgica do HU-UNIVASF foi formado por homens jovens, com lesão decorrente de ferimento por arma branca, levando a hemopneumotórax, sendo o hemitórax esquerdo mais acometido, resultando em um tempo médio de cinco dias com dreno de tórax.

\section{REFERÊNCIAS}

1. Broska Júnior CA, Botelho AB, Linhares AC, DeOliveria MS, Veronese G, Naufel Júnior CR et al. Profile of thoracic trauma victims submitted to chest drainage. Rev. Col. Bras. Cir. [Internet]. 2017 Fev [citado 2020 Abr 16]; 44(1): 27-32. Disponível em: http://www.scielo.br/scielo.php?script=sci_arttext $\& \mathrm{pid}=$ S0100-69912017000100027\&lng=en. http://dx.doi.org/10.1590/0100-69912017001005.

2. Ministério da Saúde (BR). Secretaria de Vigilância em Saúde. Departamento de Vigilância de Doenças e Agravos não Transmissíveis e Promoção da Saúde. Uma análise da situação de saúde e das causas externas. Brasília: Ministério da Saúde [Internet]. 2015[citado 2020 Jun 05]. Disponível em: https://saudebrasil.saude.gov.br/

3. Melo GBT, Alves SV, Lima MLC. Mortalidade por causas externas em Pernambuco, 2001-2003 e 2011-2013. Rev. Bras. Enferm. [Internet]. 2015 Out [citado 2021 Jan 08]; 68(5): 855-861. Disponível em: http://www.scielo.br/scielo.php?script=sci_arttext \&pid=S0034-71672015000500855\&lng=pt. https://doi.org/10.1590/0034-7167.2015680513i.

4. Frigo J, Kolhs M, Ascari RA, Machado CA, Brites J. Trauma Torácico: Análise da População Atendida em um Hospital Público de Referência no Trauma. Rev enferm UFPE on line. [Internet]. 2013[citado 2020 Dez 10];7(8):5305-12. Disponível em: https://periodicos.ufpe.br/revistas/revistaenfermag em/article/view/11807. https://doi.org/10.5205/1981-8963v7i8a11807p5305-5312-2013.

5. Rodrigues MS, Galvão IM, Santana LF. Utilização do ABCDE no atendimento do traumatizado. Rev Med [Internet]. 2017[citado 2020 Out 12]; 96(4):278-280. Disponível em: https://www.revistas.usp.br/revistadc/article/view/ 
123390. https://doi.org/10.11606/issn.16799836.v96i4p278-280

6. Westphal FL, Lima LC de, Lima Netto JC, Silva J $\mathrm{S}$ da, Santos Júnior VL dos, Westphal DC. Trauma torácico: análise de 124 pacientes submetidos à toracotomia. Rev. Col. Bras. Cir. [Internet]. 2009 Dec [citado 2020 Nov 16]; 36(6): 482-486. Disponível em: http://www.scielo.br/scielo.php?script=sci_arttext \&pid=S0100-69912009000600004\&lng=en. http://dx.doi.org/10.1590/S010069912009000600004.

7. Cipriano FG, Dessote LU. Drenagem pleural. Medicina (Ribeirão Preto) [Internet]. 2011 [citado 2020 Set 08];44(1):70-8. Disponível em: https://www.revistas.usp.br/rmrp/article/view/473 38. https://doi.org/10.11606/issn.21767262.v44i1p70-78.

8. Machado MGR. Fisioterapia no Pré e PósOperatório de Cirurgia Torácica. In: Machado MGR. Bases da Fisioterapia Respiratória Terapia Intensiva e Reabilitação. Rio de Janeiro: Guanabara Koogan; 2015. 363-376.

9. Nóbrega KCC, Pereira JVM, Costa DS. Intervenção Fisioterapêutica em Casos de pacientes Admitidos por Trauma Torácico: um Estudo Retrospectivo. Est. Cient. (UNIFAP) [Internet]. 2012[citado 2020 Dez 15]; 2(1):43-54. Disponível: https://periodicos.unifap.br/index.php/estacao/arti cle/view/458.

10. Cuba RMBF, Bezerra JAF. Traumatismo Torácico: Estudo Retrospectivo de 168 Casos. Rev. Col.
Bras. Cir. [Internet]. 2005 Abr [citado 2020 Jul 20]; 32(2):57-59. Disponível em: http://www.scielo.br/scielo.php?script=sci_arttext $\& \mathrm{pid}=$ S0100-69912005000200002\&lng=en. http://dx.doi.org/10.1590/S010069912005000200002.

11. Mendes CA, Hirano ES. Fatores Preditores de Complicações da Drenagem Torácica em Pacientes Traumatizados. Rev. Col. Bras. Cir. [Internet] 2018 [citado 2020 Out 12]; 45(2): e1543. Disponível em: https://www.scielo.br/pdf/rcbc/v45n2/pt_18094546-rcbc-45-02-e1543.pdf. https://doi.org/10.1590/0100-6991e-20181543.

12. Duarte MP, Rosa LLD, Pinheiro EM, Alves IK, Andrade ADB, Azevedo MV et al. Influência da Fisioterapia na Reabilitação de Pacientes Submetidos à Drenagem Torácica em um Hospital de Urgência e Emergência da Amazônia Legal. Rev. Elect. Ac. Sal. [Internet] 2020 [citado 2020 Out 15]; e2959. Disponível em: https://www.researchgate.net/publication/3405676 29 Influencia da fisioterapia na reabilitacao de _pacientes_submetidos_a_drenagem_toracica_em um hospital de urgencia e emergencia da am azonia_legal. https://doi.org/10.25248/reas.e2959.2020.

13. Naufel Júnior CR, Talini C, Barbier Neto L. Perfil dos Pacientes Vítimas de Trauma Torácico Atendidos no Hospital Universitário Evangélico de Curitiba (HUEC). Rev. Med. UFPR [Internet]. 2014 [citado 2020 Out 19]; 1(2):42-46. Disponível em:

https://revistas.ufpr.br/revmedicaufpr/article/view/ 40701.

http://dx.doi.org/10.5380/rmu.v1i2.40701.g25053. 